Research Article

\title{
Serre's Reduction and the Smith Forms of Multivariate Polynomial Matrices
}

\author{
Dongmei Li (iD) and Rui Liang (iD) \\ School of Mathematics and Computations, Hunan University of Science and Technology, Xiangtan 411201, Hunan, China \\ Correspondence should be addressed to Dongmei Li; dmli@hnust.edu.cn
}

Received 15 October 2019; Revised 3 March 2020; Accepted 8 April 2020; Published 13 May 2020

Academic Editor: Sang-Bing Tsai

Copyright (c) 2020 Dongmei Li and Rui Liang. This is an open access article distributed under the Creative Commons Attribution License, which permits unrestricted use, distribution, and reproduction in any medium, provided the original work is properly cited.

\begin{abstract}
The equivalence of systems plays a critical role in multidimensional systems, which are usually represented by the multivariate polynomial matrices. The Smith form of a matrix is one of the important research contents in polynomial matrices. This paper mainly investigates the Smith forms of some multivariate polynomial matrices. We have obtained several new results and criteria on the reduction of a given multivariate polynomial matrix to its Smith form. These criteria are easily checked by computing the minors of lower order of the given matrix.
\end{abstract}

\section{Introduction}

The subject of multidimensional $(n \mathrm{D})$ systems is concerned with a mathematical framework for tackling a broad range of paradigms whose analysis or synthesis requires the use of functions and polynomials in several complex variables. Many physical systems, multiple-input multiple-output systems, data analysis procedures, and learning algorithms have a natural $n \mathrm{D}$ structure due to the presence of one spatial variable. So the theory of $n \mathrm{D}$ systems is widely applied in areas of image processing, linear multipass processes, geophysical exploration, iterative learning control systems, etc. [1-9]. The equivalence of systems is one important research problem in the $n \mathrm{D}$ system theory. It is often required to transform a given system into a simpler but equivalent form. As we all know, a multivariate $(n \mathrm{D})$ polynomial matrix is often used to represent an $n \mathrm{D}$ system. So the equivalence problem of $n \mathrm{D}$ system is often transformed into the equivalence problem of $n \mathrm{D}$ polynomial matrices. For $1 \mathrm{D}$ systems, the equivalence problem has been solved $[5,7]$ by the quite mature theory of $1 \mathrm{D}$ polynomial matrix. For $n \mathrm{D}$ $(n \geq 2)$ case, since the equivalence problem is equivalent to a highly difficult problem, the isomorphism problem of two finitely presented modules, there is no hope that the equivalence problem can be solved completely. There exist two primary problems on equivalence of multivariate $(n \mathrm{D})$ polynomial matrices: one is to reduce an $n \mathrm{D}$ polynomial matrix to its Smith form. Kung et al. have obtained some interesting results about the equivalence of $n \mathrm{D}$ polynomial matrices to their Smith forms [5, 6, 10-13]. Furthermore, the Smith forms of some $n \mathrm{D}$ polynomial matrices can be computed by Maple [14]. The other is called Serre's reduction problem. One of the motivations for doing Serre's reduction for an $n \mathrm{D}$ polynomial matrix is to reduce an $n \mathrm{D}$ system to an equivalent system containing fewer equations and unknowns. Cluzeau et al. have studied Serre's reduction and presented some new interesting results in $[15,16]$.

The following problem, proposed by Serre in 1960s, plays an important role in the research problems of $n \mathrm{D}$ systems. It is not only the problem of reducing a matrix to its Smith form, but also Serre's reduction problem.

Problem 1. When is an $n \mathrm{D}(n \geq 2)$ polynomial matrix $F(z)$ equivalent to the matrix

$$
S(z)=\left(\begin{array}{cc}
I_{l-1} & 0_{l-1,1} \\
0_{1, l-1} & d
\end{array}\right),
$$

where $d=\operatorname{det} F(z)$ ? $I_{l-1}$ is the $(l-1) \times(l-1)$ identity matrix and $0_{l, m}$ is the $l \times m$ zero matrix. 
For the real number field $\mathbb{R}$, Lin et al. [12] have investigated Problem 1 for $F(z) \in \mathbb{R}^{l \times l}[z]$ with $\operatorname{det} F(z)=z_{1}-$ $f\left(z_{2}, \ldots, z_{n}\right)$ and proved that $F(z)$ is equivalent to its Smith form. Li et al. [17] have investigated Problem 1 for $F(z) \in \mathbb{R}^{l \times l}[z]$ with $\operatorname{det} F(z)=\left(z_{1}-f\left(z_{2}, \ldots, z_{n}\right)\right)^{q}$ and obtained that $F(z)$ is equivalent to its Smith form with $F(z)$ satisfying some criteria. Cluzeau et al. [13-16] also studied it and gave some new interesting results.

In this paper, we will investigate Problem 1 for the case of $F(z) \in K^{l \times l}[z] \quad$ and $\operatorname{det} \quad F(z)=\left(z_{1}-f_{1}\left(z_{2}, \ldots\right.\right.$, $\left.\left.z_{n}\right)\right)^{q_{1}}\left(z_{2}-f_{2}\left(z_{3}, \ldots, z_{n}\right)\right)^{q_{2}}$, where $q_{1}, q_{2}$ are nonnegative integers, $K$ is an arbitrary field (even a function field or a finite field). Then, we investigate the Smith forms of some rectangular polynomial matrices and consider the following problem.

Problem 2. Let $F(z) \in K^{l \times m}[z](l \leq m)$ with $d(F(z))=\left(z_{1}-\right.$ $\left.f\left(z_{2}, \ldots, z_{n}\right)\right)^{q_{1}} \cdot\left(z_{2}-f_{2}\left(z_{3}, \ldots, z_{n}\right)\right)^{q_{2}}$, where $d(F(z))$ is the greatest common divisor of the $l \times l$ minors of $F(z)$, $q_{1}, q_{2}$ are nonnegative integers. When is $F(z)$ equivalent to its Smith form?

The paper is organized as follows. In Section 2, we give some basic concepts on the equivalence of $n \mathrm{D}$ polynomial matrices. In Section 3, main results and some tractable criteria on equivalence of several kinds of polynomial matrices are proposed. In Section 4, an example is provided to illustrate the effectiveness of our constructive method.

\section{Preliminaries}

In the following, $K[z]=K\left[z_{1}, z_{2}, \ldots, z_{n}\right]$ will denote the polynomial ring in $n$ variables $z_{1}, z_{2}, \ldots, z_{n}$ with coefficients in arbitrary field $K, \bar{K}$ will be an algebraic closed field of $K$, $\bar{K}^{n}$ will be the $n$ dimensional vector space over $\bar{K}, K^{l \times m}[z]$ will denote the set of $l \times m$ matrices with their entries in $K[z], K^{l \times m}\left[z_{2}, \ldots, z_{n}\right]$ will denote the set of $l \times m$ matrices with their entries in $K\left[z_{2}, \ldots, z_{n}\right]$, the $r \times r$ identity matrix will be denoted by $I_{r}$ and the $r \times t$ zero matrix will be denoted by $0_{r, t}$. A matrix over $K[z]$ with its determinant in $K$ is said to be unimodular. Throughout the paper, the argument $(z)$ is omitted whenever its omission does not cause confusion.

Definition 1 (see [18]).Let $F(z) \in K^{l \times m}[z]$ be of full row (column) rank. Then $F(z)$ is said to be zero left prime (zero right prime) if the $l \times l(m \times m)$ minors of $F(z)$ generate the unit ideal $K[z]$.

If $F(z) \in K^{l \times m}[z]$ is zero left prime (zero right prime), then $F(z)$ is called simply to be ZLP (ZRP). According to the Quillen-Suslin theorem [19], we have that $F(z)$ is ZLP if and only if there is a matrix $N(z) \in K^{m \times m}[z]$ such that $F(z) \cdot N(z)=\left(I_{l} 0_{l \times(m-l)}\right)$. It is also equivalent to say that any ZLP (ZRP) matrix over $K[z]$ can be completed to an unimodular (invertible) matrix.

Definition 2 Let $F(z) \in K^{l \times m}[z], l \leq m$, and $\Phi_{i}$ be polynomially defined as follows:

$$
\Phi_{i}= \begin{cases}d_{i}(F) / d_{i-1}(F), & 1 \leq i \leq r, \\ 0, & r<i \leq l,\end{cases}
$$

where $r$ is the rank of $F(z), d_{0}(F) \equiv 1, d_{i}(F)$ is the greatest common divisor of the $i \times i$ minors of $F(z)$, and $\Phi_{i}$ satisfies $\Phi_{i} \mid \Phi_{i+1}, i=1, \ldots, l$. Then, we define the Smith form of $F(z)$ as

$$
S=\left(\operatorname{diag}\left\{\Phi_{i}\right\} \quad 0_{l \times(m-l)}\right) .
$$

Definition 3 (see [17]). Let $T_{1}(z)$ and $T_{2}(z)$ denote two matrices in $K^{l \times m}[z], T_{1}(z)$, and $T_{2}(z)$ are said to be equivalent if there exist two invertible matrices $M(z) \in K^{l \times l}[z]$ and $N(z) \in K^{m \times m}[z]$ such that $T_{2}(z)=M(z) T_{1}(z) N(z)$.

\section{Main Results}

In this section, the main results are presented. First, we give some well-known results and provide an answer to Problem 1 for case of $\operatorname{det} F(z)=\left(z_{1}-f_{1}\left(z_{2}, \ldots, z_{n}\right)\right)\left(z_{2}-f_{2}\right.$ $\left.\left(z_{3}, \ldots, z_{n}\right)\right)$ in Subsection 3.1. Then we extend this result to more general case of $\operatorname{det} F(z)=\left(z_{1}-f_{1}\left(z_{2}, \ldots, z_{n}\right)\right)$ $\left(z_{2}-f_{2}\left(z_{3}, \ldots, z_{n}\right)\right)^{q_{2}}$ and present a complete answer to Problem 2 in Subsection 3.2.

3.1. Equivalent Theorem. In order to prove our main results, we first give several useful lemmas.

Lemma 1 (see [20]). Let $f_{1}(z), \ldots, f_{s}(z) \in K[z]$, then $f_{1}(z), \ldots, f_{s}(z)$ have no common zeros in $\bar{K}^{n}$ (are zero coprime) if and only if there exist $u_{1}(z), \ldots, u_{s}(z) \in K[z]$ such that

$$
u_{1}(z) f_{1}(z)+\cdots+u_{s}(z) f_{s}(z)=1,
$$

i.e., $f_{1}(z), \ldots, f_{s}(z)$ is a ZLP row vector in $K^{1 \times s}[z]$, or $f_{1}(z), \ldots, f_{s}(z)$ generate the unit ideal $K[z]$.

Lemma 2 (see [20]). Let $g(z) \in K\left[z_{1}, z_{2}, \ldots, z_{n}\right]$ and $f\left(z_{2}, \ldots, z_{n}\right) \in K\left[z_{2}, \ldots, z_{n}\right]$. Suppose that $g\left(f\left(z_{2}, \ldots\right.\right.$, $\left.\left.z_{n}\right), z_{2}, \ldots, z_{n}\right)=0$, then $z_{1}-f\left(z_{2}, \ldots, z_{n}\right)$ is a divisor of $g(z)$.

Lemma 3 (see [17]). Let $F(z), F_{1}(z), F_{2}(z) \in K^{l \times l}[z]$, $F(z)=F_{1}(z) \cdot F_{2}(z)$. If the $(l-1) \times(l-1)$ minors of $F(z)$ have no common zeros in $\bar{K}^{n}$ (generate $K[z]$ ), then the $(l-1) \times(l-1)$ minors of $F_{i}(z)$ have no common zeros in $\bar{K}^{n}$ (generate $K[z])$ for $i=1,2$.

Proof. The proof is similar to that of Lemma 2.2 in [17], so it is omitted here.

Lemma 4 (see [18]). Let $Q \in K^{(l-1) \times l}[z]$ be of normal full rank, if the reduced minors of $Q$ generate $K[z]$, then there exists a ZLP matrix $w \in K^{l \times 1}[z]$ such that $Q \cdot w=0_{(l-1), 1}$.

The following result is presented in [20], for the convenience of the reader, we record it here. 
Lemma 5 (see [20]). Let $F(z)=F\left(z_{1}, z_{2}, \ldots, z_{n}\right) \in K^{l \times l}[z]$, $f\left(z_{2}, \ldots, z_{n}\right) \in K\left[z_{2}, \ldots, z_{n}\right]$, if the $(l-1) \times(l-1)$ minors of $F(z)$ generate $K[z]$, then the $(l-1) \times(l-1)$ minors of $F\left(f\left(z_{2}, \ldots, z_{n}\right), z_{2}, \ldots, z_{n}\right)$ also generate $K[z]$.

Proof. Assume that the $(l-1) \times(l-1)$ minors of $F(z)$ are $c_{1}\left(z_{1}, \ldots, z_{n}\right), \ldots, c_{r}\left(z_{1}, \ldots, z_{n}\right)$. If the $(l-1) \times(l-1)$ minors of $F(z)$ generate $K[z]$, then there exist $u_{1}\left(z_{1}, z_{2}, \ldots, z_{n}\right), \ldots, u_{r}\left(z_{1}, z_{2}, \ldots, z_{n}\right) \in K[z]$ such that

$$
\begin{aligned}
& u_{1}\left(z_{1}, z_{2}, \ldots, z_{n}\right) c_{1}\left(z_{1}, z_{2}, \ldots, z_{n}\right)+\cdots \\
& \quad+u_{r}\left(z_{1}, z_{2}, \ldots, z_{n}\right) c_{r}\left(z_{1}, \ldots, z_{n}\right)=1 .
\end{aligned}
$$

Substitute $z_{1}=f\left(z_{2}, \ldots, z_{n}\right)$, then

$$
\begin{aligned}
& u_{1}\left(f\left(z_{2}, \ldots, z_{n}\right), z_{2}, \ldots, z_{n}\right) c_{1}\left(f\left(z_{2}, \ldots, z_{n}\right), z_{2}, \ldots, z_{n}\right) \\
& \quad+\cdots+u_{r}\left(f\left(z_{2}, \ldots, z_{n}\right), z_{2}, \ldots, z_{n}\right) c_{r} \\
& \quad\left(f\left(z_{2}, \ldots, z_{n}\right), z_{2}, \ldots, z_{n}\right)=1 .
\end{aligned}
$$

It is straightforward that $c_{1}\left(f\left(z_{2}, \ldots, z_{n}\right)\right.$, $\left.z_{2}, \ldots, z_{n}\right) c_{r}\left(f\left(z_{2}, \ldots, z_{n}\right), z_{2}, \ldots, z_{n}\right)$ are the $(l-1) \times(l-$ 1) minors of $F\left(f\left(z_{2}, \ldots, z_{n}\right), z_{2}, \ldots, z_{n}\right)$. Thus the $(l-1) \times$ $(l-1)$ minors of $F\left(f\left(z_{2}, \ldots, z_{n}\right), z_{2}, \ldots, z_{n}\right)$ also generate $K[z]$.

Lemma 6. Let $F(z) \in K^{l \times l}[z]$, then $\operatorname{det} F(z)$ and the $(l-$ $1) \times(l-1)$ minors of $F(z)$ have no common zeros if and only if the $(l-1) \times(l-1)$ minors of $F(z)$ have no common zeros.

Proof. Necessity. Assuming that the $(l-1) \times(l-1)$ minors of $F(z)$ have a common zero point $a_{0}$, by Laplace expansion,

we can easily know that $a_{0}$ is also a zero point of $\operatorname{det} F(z)$, contradicting the fact that $\operatorname{det} F(z)$ and the $(l-1) \times(l-1)$ minors of $F(z)$ have no common zeros. Thus, the $(l-1) \times$ $(l-1)$ minors of $F(z)$ have no common zeros.

Sufficiency. It is straightforward.

For the convenience, we first define $P_{1}(z), P_{2}(z), P(z)$ as follows:

$$
\begin{aligned}
P_{1}(z) & =\left(\begin{array}{cc}
I_{l-1} & 0_{l-1,1} \\
0_{1, l-1} & z_{1}-f_{1}\left(z_{2}, \ldots, z_{n}\right)
\end{array}\right), \\
P_{2}(z) & =\left(\begin{array}{cc}
I_{l-1} & 0_{l-1,1} \\
0_{1, l-1} & z_{2}-f_{2}\left(z_{3}, \ldots, z_{n}\right)
\end{array}\right), \\
P(z) & =\left(\begin{array}{cc}
I_{l-1} & 0_{l-1,1} \\
0_{1, l-1} & \left(z_{1}-f_{1}\left(z_{2}, \ldots, z_{n}\right)\right)\left(z_{2}-f_{2}\left(z_{3}, \ldots, z_{n}\right)\right)
\end{array}\right) .
\end{aligned}
$$

We have the following key conclusion which is very important to derive our main results.

Lemma 7. Let $F(z) \in K^{l \times l}[z]$ and $F(z)=P_{1}(z) V(z) P_{2}(z)$, where $V(z) \in K^{l \times l}\left[z_{2}, \ldots, z_{n}\right]$ is unimodular. If all the $(l-$ $1) \times(l-1)$ minors of $F(z)$ generate $K[z]$, then $F(z)$ is equivalent to its Smith form $P(z)$.

Proof. We prove this by induction on $l$. When $l=2$, let

$$
V(z)=\left(\begin{array}{ll}
v_{11} & v_{12} \\
v_{21} & v_{22}
\end{array}\right)
$$

then

$$
F(z)=\left(\begin{array}{cc}
v_{11} & v_{12} \cdot\left(z_{2}-f_{2}\left(z_{3}, \ldots, z_{n}\right)\right) \\
v_{21} \cdot\left(z_{1}-f_{1}\left(z_{2}, \ldots, z_{n}\right)\right) & v_{22} \cdot\left(z_{1}-f_{1}\left(z_{2}, \ldots, z_{n}\right)\right)\left(z_{2}-f_{2}\left(z_{3}, \ldots, z_{n}\right)\right)
\end{array}\right) .
$$

Obviously, $\operatorname{det} F(z)=c \cdot\left(z_{1}-f_{1}\left(z_{2}, \ldots, z_{n}\right)\right)\left(z_{2}-f_{2}\right.$ $\left.\left(z_{3}, \ldots, z_{n}\right)\right), 0 \neq c \in K$. Note that $V(z)$ is unimodular, then $v_{11}, v_{12}$ have no common zeros. Assume that $v_{11}, v_{12}\left(z_{2}-\right.$ $\left.f_{2}\left(z_{3}, \ldots, z_{n}\right)\right)$ have a zero $\alpha_{0}=\left(z_{20}, z_{30}, \ldots, z_{n 0}\right)$, where $z_{20}=f_{2}\left(z_{30}, \ldots, z_{n 0}\right)$, then $v_{11}, v_{12} \cdot\left(z_{2}-f_{2}\left(z_{3}, \ldots, z_{n}\right)\right)$, $v_{21} \cdot\left(z_{1}-f_{1}\left(z_{2}, \ldots, z_{n}\right)\right), v_{22} \cdot\left(z_{1}-f_{1}\left(z_{2}, \ldots, z_{n}\right)\right)\left(z_{2}-f_{2}\right.$ $\left.\left(z_{3}, \ldots, z_{n}\right)\right)$ has a common zero $\left(f_{1}\left(\alpha_{0}\right), z_{20}, z_{30}, \ldots, z_{n o}\right)$. This is a contradiction. Thus, $v_{11}, v_{12} \cdot\left(z_{2}-f_{2}\left(z_{3}, \ldots, z_{n}\right)\right)$ have no common zeros, and $\left(v_{11}, v_{12} \cdot\left(z_{2}-f_{2}\left(z_{3}, \ldots, z_{n}\right)\right)\right)$ is a unimodular row. According to Quillen-Suslin Theorem, there exists a $2 \times 2$ unimodular matrix $M_{1}(z) \in K^{2 \times 2}[z]$ such that

$$
\left(v_{11}, v_{12}\left(z_{2}-f_{2}\left(z_{3}, \ldots, z_{n}\right)\right)\right) M_{1}(z)=(1,0) .
$$

So

$$
F(z) M_{1}(z)=\left(\begin{array}{cc}
1 & 0 \\
p_{1}(z) & p_{2}(z)
\end{array}\right)
$$

where $p_{1}(z), p_{2}(z) \in K[z]$. Then there exists the unimodular matrix $M_{2}(z)=\left(\begin{array}{cc}1 & 0 \\ -p_{1}(z) & 1\end{array}\right)$ such that

$$
M_{2}(z) F(z) M_{1}(z)=\left(\begin{array}{cc}
1 & 0 \\
0 & p_{2}(z)
\end{array}\right) \text {. }
$$

Note that $p_{2}(z)=\operatorname{det}\left(M_{2}(z) F(z) M_{1}(z)\right)=u \cdot \operatorname{det} F(z)=$ $u^{\prime} \cdot\left(z_{1}-f_{1}\left(z_{2}, \ldots, z_{n}\right)\right)\left(z_{2}-f_{2}\left(z_{3}, \ldots, z_{n}\right)\right), \quad 0 \neq u, u^{\prime} \in K$. Thus, $F(z)$ is equivalent to its Smith form

$$
P(z)=\left(\begin{array}{lc}
1 & 0 \\
0 & \left(z_{1}-f_{1}\left(z_{2}, \ldots, z_{n}\right)\right)\left(z_{2}-f_{2}\left(z_{3}, \ldots, z_{n}\right)\right)
\end{array}\right) .
$$

So the conclusion is true for $l=2$.

Assume that the conclusion is true for $l-1$, we investigate the case of $l$. Let $V(z)=\left(\begin{array}{ll}V_{11} & V_{12} \\ V_{21} & V_{22}\end{array}\right)$, where $V_{11} \in K^{(l-1) \times(l-1)}\left[z_{2}, \ldots, z_{n}\right], \quad V_{12} \in K^{(l-1) \times 1}\left[z_{2}, \ldots, z_{n}\right]$, $V_{21} \in K^{1 \times(l-1)}\left[z_{2}, \ldots, z_{n}\right], V_{22} \in K^{1 \times 1}\left[z_{2}, \ldots, z_{n}\right]$. Then 


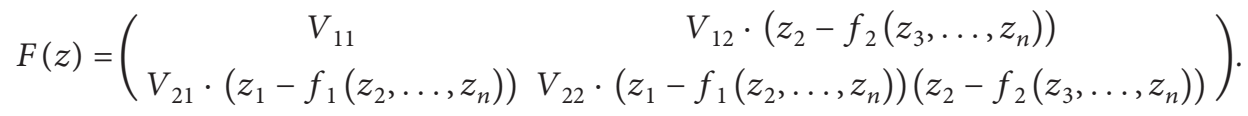

Let $\left(v_{11}, \ldots, v_{1, l-1}, v_{1 l}\right)$ be the first row of $V(z)$. Since $V(z)$ is unimodular, using Laplace Theorem and expanding its first row, we obtain that $v_{11} a_{1}(z)+\cdots+v_{1, l-1} a_{l-1}(z)+$ $v_{1 l} a_{l}(z)=k$, where $a_{1}(z), \ldots, a_{l-1}(z), a(z)$ are all the $(l-$ $1) \times(l-1)$ minors of $V(z)$ and $0 \neq k \in K$. Combined with Lemma 1 , we have that $v_{11}, \ldots, v_{1, l-1}, v_{1 l}$ have no common zeros. Assume that $v_{11}, \ldots, v_{1, l-1}, v_{1 l}\left(z_{2}-f_{2}\left(z_{3}, \ldots, z_{n}\right)\right)$ have a common zero $\alpha_{0}=\left(z_{20}, z_{30}, \ldots, z_{n 0}\right)$, then all the $(l-$ $1) \times(l-1)$ minors of $F(z)$ have a common zero $\left(f_{1}\left(\alpha_{0}\right)\right.$, $\left.z_{20}, z_{30}, \ldots, z_{n 0}\right)$. This is a contradiction. Thus, $\left(v_{11}, \ldots\right.$, $\left.v_{1, l-1}, v_{1 l}\left(z_{2}-f_{2}\left(z_{3}, \ldots, z_{n}\right)\right)\right)$ is a unimodular row in $K\left[z_{2}, \ldots, z_{n}\right]$, by the Quillen-Suslin Theorem, there exists a unimodular matrix $M_{1}(z)$ such that

$$
\left(v_{11}, \ldots, v_{1, l-1}, v_{1 l}\left(z_{2}-f_{2}\left(z_{3}, \ldots, z_{n}\right)\right)\right) M_{1}(z)=(1,0, \ldots, 0) .
$$

So

$$
F(z) M_{1}(z)=\left(\begin{array}{cc}
1 & 0_{1, l-1} \\
Q_{1}(z) & Q_{2}(z)
\end{array}\right)
$$

where $Q_{1}(z) \in K^{(l-1) \times 1}[z], \quad Q_{2}(z) \in K^{(l-1) \times(l-1)}[z]$. Then there exists the matrix

$$
M_{2}(z)=\left(\begin{array}{cc}
1 & 0_{1, l-1} \\
-Q_{1}(z) & I_{l-1}
\end{array}\right)
$$

such that

$$
M_{2}(z) F(z) M_{1}(z)=\left(\begin{array}{cc}
1 & 0_{1, l-1} \\
0_{l-1,1} & Q_{2}(z)
\end{array}\right) .
$$

Setting $N(z)=M_{2}(z) F(z) M_{1}(z)$, since all the $(l-1) \times$ $(l-1)$ minors of $F(z)$ have no common zeros, combined with Lemma 3 , we have that all the $(l-1) \times(l-1)$ minors of $N(z)$ have no common zeros. Note that an $(l-1) \times(l-$ 1) minor of $N(z)$ is just an $(l-2) \times(l-2)$ minors of $Q_{2}(z)$ or $\operatorname{det} Q_{2}(z)$ or 0 , then $\operatorname{det} Q_{2}(z)$ and the $(l-2) \times(l-2)$ minors of $Q_{2}(z)$ have no common zeros. By Lemma 6, the $(l-2) \times(l-2)$ minors of $Q_{2}(z)$ have no common zeros. By the inductive hypothesis, there exist two $(l-1) \times(l-1)$ unimodular matrices $N_{1}(z), N_{2}(z)$ such that

$$
\begin{aligned}
& N_{2}(z) Q_{2}(z) N_{1}(z) \\
& \quad=\left(\begin{array}{cc}
I_{l-2} & 0_{l-2,1} \\
0_{1, l-2} & \left(z_{1}-f_{1}\left(z_{2}, \ldots, z_{n}\right)\right)\left(z_{2}-f_{2}\left(z_{3}, \ldots, z_{n}\right)\right)
\end{array}\right),
\end{aligned}
$$

then

$$
\begin{aligned}
& \left(\begin{array}{cc}
1 & 0_{1, l-1} \\
0_{l-1,1} & N_{2}(z)
\end{array}\right) N(z, w)\left(\begin{array}{cc}
1 & 0_{1, l-1} \\
0_{1, l-1} & N_{1}(z)
\end{array}\right) \\
& \quad=\left(\begin{array}{cc}
1 & 0_{1, l-1} \\
0_{l-1,1} & N_{2}(z) Q_{2}(z) N_{1}(z)
\end{array}\right) \\
& \quad=\left(\begin{array}{cc}
I_{l-1} & 0_{l-1,1} \\
0_{1, l-1} & \left(z_{1}-f_{1}\left(z_{2}, \ldots, z_{n}\right)\right)\left(z_{2}-f_{2}\left(z_{3}, \ldots, z_{n}\right)\right)
\end{array}\right) .
\end{aligned}
$$

Thus, $N(z)$ is equivalent to the matrix $P(z)$, combined with $F(z)$ is equivalent to the matrix $N(z)$; then we obtain that $F(z)$ is equivalent to the matrix $P(z)$, and $P(z)$ is the Smith form of $F(z)$.

Now we are going to state one of our main results, which give partial answer to Problem 1. We recall the notation

$$
P(z)=\left(\begin{array}{cc}
I_{l-1} & 0_{l-1,1} \\
0_{1, l-1} & \left(z_{1}-f_{1}\left(z_{2}, \ldots, z_{n}\right)\right)\left(z_{2}-f_{2}\left(z_{3}, \ldots, z_{n}\right)\right)
\end{array}\right) .
$$

Theorem 1. Let $F(z) \in K^{l \times l}[z]$ with $\operatorname{det} F(z)=\left(z_{1}-\right.$ $\left.f_{1}\left(z_{2}, \ldots, z_{n}\right)\right)\left(z_{2}-f_{2}\left(z_{3}, \ldots, z_{n}\right)\right) . F(z)$ is equivalent to its Smith form $P(z)$ if and only if all the $(l-1) \times(l-1)$ minors of $F(z)$ generate $K[z]$.

Proof. Sufficiency. Because $\operatorname{det} F\left(z_{1}, f_{2}\left(z_{3}, \ldots\right.\right.$, $\left.\left.z_{n}\right), z_{3}, \ldots, z_{n}\right)=0$, then the rank of $F\left(z_{1}, f_{2}\left(z_{3}, \ldots, z_{n}\right)\right.$, $\left.z_{3}, \ldots, z_{n}\right) \leq l-1$. Since all the $(l-1) \times(l-1)$ minors of $F(z)$ generate $K[z]$, by Lemma 5 , the $(l-1) \times(l-1) \mathrm{mi}-$ nors of $F\left(z_{1}, f_{2}\left(z_{3}, \ldots, z_{n}\right), z_{3}, \ldots, z_{n}\right)$ also generate $K[z]$, then $\operatorname{rank} F\left(z_{1}, f_{2}\left(z_{3}, \ldots, z_{n}\right), z_{3}, \ldots, z_{n}\right)=l-1$ for every $\left(z_{1}, z_{3}, \ldots, z_{n}\right) \in \bar{K}$. By Lemma 4 , there exists a ZRP column vector $Y_{1}(z) \in K^{l \times 1}[z]$ such that

$$
F\left(z_{1}, f_{2}\left(z_{3}, \ldots, z_{n}\right), z_{3}, \ldots, z_{n}\right) Y_{1}(z) \equiv(0, \ldots, 0)^{T} .
$$

By the Quillen-Suslin theorem, an $l \times l$ unimodular matrix $U_{11}(z)$ can be constructed such that $Y_{1}(z)$ is its last column. Then the elements of the last column of $F\left(z_{1}, f_{2}\left(z_{3}, \ldots, z_{n}\right), z_{3}, \ldots, z_{n}\right) \cdot U_{11}(z)$ are zero polynomials. By Lemma 2, the last column of $F(z) \cdot U_{11}(z)$ have the common divisor $z_{2}-f_{2}\left(z_{3}, \ldots, z_{n}\right)$, i.e.,

$$
F(z) \cdot U_{11}(z)=F_{1}(z) \cdot\left(\begin{array}{cc}
I_{l-1} & 0_{l-1,1} \\
0_{1, l-1} & z_{2}-f_{2}\left(z_{3}, \ldots, z_{n}\right)
\end{array}\right),
$$

for some $F_{1}(z) \in K^{l \times l}[z]$. Let $U_{1}(z)=U_{11}^{-1}(z)$, then we have

$$
F(z)=F_{1}(z) P_{2}(z) U_{1}(z) \text {. }
$$


Note that $U_{1}(z), U_{11}(z)$ are unimodular, and $\operatorname{det} F(z)=\left(z_{1}-f_{1}\left(z_{2}, \ldots, z_{n}\right)\right)\left(z_{2}-f_{2}\left(z_{3}, \ldots, z_{n}\right)\right)$, then

$\operatorname{det} F_{1}(z)=c \cdot\left(z_{1}-f_{1}\left(z_{2}, \ldots, z_{n}\right)\right), \quad 0 \neq c \in K$.

From Lemma 3, we have that the $(l-1) \times(l-1)$ minors of $F_{1}(z)$ also generate $K[z]$. Note that $\operatorname{det} F_{1}\left(f_{1}\left(z_{2}, \ldots, z_{n}\right), z_{2}, z_{3}, \ldots, z_{n}\right)=0$, combined with Lemma 5, we obtain that the $(l-1) \times(l-1)$ minors of $F_{1}\left(f_{1}\left(z_{2}, \ldots, z_{n}\right), z_{2}, z_{3}, \ldots, z_{n}\right)$ also generate $K[z]$ for $\left(z_{2}, z_{3}, \ldots, z_{n}\right) \in \bar{K}$. Similarly, a unimodular matrix $V_{11}(z) \in K^{l \times l}\left[z_{2}, \ldots, z_{n}\right]$ can be constructed such that

$$
F_{1}(z) V_{11}(z)=F_{2}(z) \cdot\left(\begin{array}{cc}
I_{l-1} & 0_{l-1,1} \\
0_{1, l-1} & z_{1}-f_{1}\left(z_{2}, \ldots, z_{n}\right)
\end{array}\right),
$$

where $F_{2}(z) \in K^{l \times l}[z]$. Let $V_{1}(z)=V_{11}^{-1}(z)$, then we have

$$
F_{1}(z)=F_{2}(z) P_{1}(z) V_{1}(z) \text {. }
$$

Note that $V_{1}(z) \in K^{l \times l}\left[z_{2}, \ldots, z_{n}\right]$ is unimodular, and $\operatorname{det} F_{1}(z)=c_{1} \cdot\left(z_{1}-f_{1}\left(z_{2}, \ldots, z_{n}\right)\right)$, then $\operatorname{det} F_{2}(z)=c_{2}, 0 \neq c_{2} \in K, F_{2}(z)$ is unimodular, and

$$
F(z)=F_{2}(z) P_{1}(z) V_{1}(z) P_{2}(z) U_{1}(z) \text {. }
$$

By Lemma 7, the matrix $P_{1}(z) V_{1}(z) P_{2}(z)$ is equivalent to $P(z)=\left(\begin{array}{cc}I_{l-1} & 0_{l-1,1} \\ 0_{1, l-1} & \left(z_{1}-f_{1}\left(z_{2}, \ldots, z_{n}\right)\right)\left(z_{2}-f_{2}\left(z_{3}, \ldots, z_{n}\right)\right)\end{array}\right)$.

Combined with $F_{2}(z), U_{1}(z)$ are unimodular, we obtain that $F(z)$ is equivalent to its Smith form $P(z)$.

Necessity. If $F(z)$ is equivalent to $P(z)$, there exist two unimodular matrices $U(z), V(z)$ such that $F(z)=V(z) P(z) U(z)$. Note that all the $(l-1) \times(l-1)$ minors of $P(z)$ have no common zeros; combined with Lemma 3, we have that all the $(l-1) \times(l-1)$ minors of $F(z)$ have no common zeros, i.e., all the $(l-1) \times(l-1)$ minors of $F(z)$ generate $K[z]$.

In the following, we will extend the above result to more general case.

\subsection{Generalization}

Lemma 8. Let $D(z) \in K^{l \times l}[z]$ with $D(z)=\left(\begin{array}{cc}I_{l-1} & 0 \\ 0 & h(z)\end{array}\right)$, $h(z) \in K[z]$, and $V(z) \in K^{l \times l}[z]$ be a unimodular matrix. If all the $(l-1) \times(l-1)$ minors of $D^{s}(z) V(z) D^{t}(z)$ generate $K[z]$ and $s, t$ are positive integers, then $D^{s}(z) V(z) D^{t}(z)$ is equivalent to $D^{s+t}(z)$.

Proof. Let

$$
V(z)=\left(\begin{array}{cc}
V_{11}(z) & V_{12}(z) \\
V_{21}(z) & V_{22}(z)
\end{array}\right)
$$

where $\quad V_{11}(z) \in K^{(l-1) \times(l-1)}[z], \quad V_{12}(z) \in K^{(l-1) \times 1}[z]$, $V_{21}(z) \in K^{1 \times(l-1)}[z], V_{22}(z) \in K^{1 \times 1}[z]$.
Then

$F(z)=D^{s}(z) V(z) D^{t}(z)=\left(\begin{array}{cc}V_{11}(z) & V_{12}(z) h^{t}(z) \\ V_{21}(z) h^{s}(z) & V_{22}(z) h^{s+t}(z)\end{array}\right)$,

and $\operatorname{det} F(z)=c \cdot h^{s+t}(z), 0 \neq c \in K$.

Next, we will proof that $\left(V_{11}(z) V_{12}(z) h^{t}(z)\right)$ is a ZLP matrix. Note that $V(z)$ is unimodular, then $\left(V_{11}(z) V_{12}(z)\right)$ is a ZLP matrix. Let $c_{1}(z), c_{2}(z), \ldots$, $c_{l}(z)$ denote all the $(l-1) \times(l-1)$ minors of $\left(V_{11}(z) V_{12}(z)\right)$. Then all the $(l-1) \times(l-1)$ minors of $\left(V_{11}(z) V_{12}(z) h^{t}(z)\right) \quad$ are $\quad c_{1}(z), c_{2}(z) h^{t}(z), \ldots$, $c_{l}(z) h^{t}(z)$. We can prove that $c_{1}(z), c_{2}(z) h^{t}(z), \ldots$, $c_{l}(z) h^{t}(z)$ have no common zeros. Suppose that $c_{1}(z)$, $c_{2}(z) h^{t}(z), \ldots, c_{l}(z) h^{t}(z)$ have a common zero $\alpha_{0}$ and combined with $c_{1}(z), c_{2}(z), \ldots, c_{l}(z)$ have no common zeros, so $\alpha_{0}$ is a zero of $c_{1}(z)$ and $h(z)$. Note that the elements of the last row of matrix $F(z)$ all have the factor $h(z)$ and that an $(l-1) \times(l-1)$ minor of $F(z)$ is just $c_{1}(z)$ or includes the factor $h(z)$, so the $(l-1) \times(l-1)$ minors of $F(z)$ have the common zero $\alpha_{0}$, this is a contradiction. Thus, all the $(l-1) \times$ $(l-1)$ minors of $\left(V_{11}(z) V_{12}(z) h^{t}(z)\right)$ have no common zeros, and it is a ZLP matrix. According to Quillen-Suslin theorem, there exists an $m \times m$ unimodular matrix $N_{1}(z)$ such that

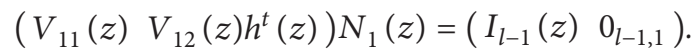

Then,

$$
F(z) N_{1}(z)=\left(\begin{array}{cc}
I_{l-1}(z) & 0_{l-1,1} \\
H_{1}(z) & H_{2}(z)
\end{array}\right),
$$

for some $H_{1}(z), H_{2}(z) \in K[z]$. Let

$$
N_{2}(z)=\left(\begin{array}{cc}
I_{l-1}(z) & 0_{l-1,1} \\
-H_{1}(z) & 1
\end{array}\right) \text {. }
$$

Then,

$$
N_{2}(z) F(z) N_{1}(z)=\left(\begin{array}{cc}
I_{l-1}(z) & 0_{l-1,1} \\
0_{1, l-1} & H_{2}(z)
\end{array}\right) .
$$

Note that $N_{1}(z), N_{2}(z)$ are unimodular, then $H_{2}(z)=u \cdot \operatorname{det} F(z)=u^{\prime} \cdot h^{s+t}(z), 0 \neq u \in K, u^{\prime}=u \cdot c$, thus $F(z)$ is equivalent to $D^{s+t}(z)$.

Now we investigate Problem 1 for the case of $q_{1}=q_{2}$. Denote

$$
P^{q}(z)=\left(\begin{array}{cc}
I_{l-1} & 0_{l-1,1} \\
0_{1, l-1} & \left(z_{1}-f_{1}\left(z_{2}, \ldots, z_{n}\right)\right)^{q}\left(z_{2}-f_{2}\left(z_{3}, \ldots, z_{n}\right)\right)^{q}
\end{array}\right) .
$$

Theorem 2. Let $F(z) \in K^{l \times l}[z]$ with $\operatorname{det} F(z)=\left(z_{1}-\right.$ $\left.f_{1}\left(z_{2}, . ., z_{n}\right)\right)^{q}\left(z_{2}-f_{2}\left(z_{3}, \ldots, z_{n}\right)\right)^{q}$, where $q$ is a positive integer. Then $F(z)$ is equivalent to its Smith form $P^{q}(z)$ if and only if all the $(l-1) \times(l-1)$ minors of $F(z)$ generate $K[z]$.

Proof. Sufficiency. Notice that $\operatorname{det} F\left(z_{1}, f_{2}\left(z_{3}, \ldots, z_{n}\right)\right.$, $\left.z_{3}, \ldots, z_{n}\right)=0$, we have that rank $F\left(z_{1}, f_{2}\left(z_{3}, \ldots, z_{n}\right)\right.$ 
$\left., z_{3}, \ldots, z_{n}\right) \leq l-1$. Since all the $(l-1) \times(l-1)$ minors of $F(z)$ generate the unit ideal $K[z]$, according to Lemma 5, then the $(l-1) \times(l-1)$ minors of $F\left(z_{1}, f_{2}\left(z_{3}, \ldots, z_{n}\right)\right.$, $\left.z_{3}, \ldots, z_{n}\right)$ also generate the unit ideal $K[z]$. Hence, $\operatorname{rank} F\left(z_{1}, f_{2}\left(z_{3}, \ldots, z_{n}\right), z_{3}, \ldots, z_{n}\right)=l-1$ for every $\left(z_{1}, z_{3}, \ldots, z_{n}\right) \in \bar{K}$, repeating the proof of Theorem 1 , we have that

$$
F(z)=F_{1}(z) P_{1}(z) V_{1}(z) P_{2}(z) U_{1}(z),
$$

where $V_{1}(z) \in K^{l \times l}\left[z_{2}, \ldots, z_{n}\right], U_{1}(z) \in K^{l \times l}[z]$ are unimodular, $\quad F_{1}(z) \in K^{l \times l}[z]$ with $\operatorname{det} F_{1}(z)=\left(z_{1}-f_{1}\right.$ $\left.\left(z_{2}, \ldots, z_{n}\right)\right)^{q-1}\left(z_{2}-f_{2}\left(z_{3}, \ldots, z_{n}\right)\right)^{q-1}$. By Lemma 3, all the $(l-1) \times(l-1)$ minors of $P_{1}(z) V_{1}(z) P_{2}(z)$ also generate $K[z]$. According to Lemma 7, there exist unimodular matrices $M_{1}(z), N_{1}(z) \in K^{l \times l}[z]$ such that $P_{1}(z) V_{1}(z)$ $P_{2}(z)=M_{1}(z) P_{1}(z) P_{2}(z) N_{1}(z)=M_{1}(z) P(z) \quad N_{1}(z)$. Thus

$$
F(z)=F_{1}(z) M_{1}(z) P(z) N_{1}(z) U_{1}(z) .
$$

Again by Lemma 3, we obtain that all the $(l-1) \times(l-1)$ minors of $F_{1}(z)$ generate $K[z]$. If $q \geq 2$, iterating the preceding process, we obtain that

$$
F_{1}(z)=F_{2}(z) M_{2}(z) P(z) N_{2}(z),
$$

where $F_{2}(z) \in K^{l \times l}[z]$ with $\operatorname{det} F_{2}(z)=\left(z_{1}-f_{1}\left(z_{2}, \ldots\right.\right.$, $\left.\left.z_{n}\right)\right)^{q-2}\left(z_{2}-f_{2}\left(z_{3}, \ldots, z_{n}\right)\right)^{q-2}$. Then

$$
F(z)=F_{2}(z) M_{2}(z) P(z) N_{2}(z) M_{1}(z) P(z) N_{1}(z) U_{1}(z) .
$$

Let $S_{2}(z)=N_{2}(z) M_{1}(z)$, we have that $S_{2}(z)$ is unimodular and all the $(l-1) \times(l-1)$ minors of $P(z) S_{2}(z) P(z)$ generate $K[z]$. By Lemma $8, P(z) S_{2}(z) P(z)$ is equivalent to $P^{2}(z)$, that is, there exist unimodular matrices $L_{1}(z), T_{1}(z) \in K^{l \times l}[z] \quad$ such that $P(z) S_{2}(z) P(z)=$ $L_{1}(z) P^{2}(z) T_{1}(z)$. Furthermore,

$$
F(z)=F_{2}(z) M_{2}(z) L_{1}(z) P^{2}(z) T_{1}(z) N_{1}(z) U_{1}(z) .
$$

Let $V_{2}(z)=M_{2}(z) L_{1}(z), U_{2}(z)=T_{1}(z) N_{1}(z) U_{1}(z)$, then $V_{2}(z), U_{2}(z)$ are unimodular, and

$$
F(z)=F_{2}(z) V_{2}(z) P^{2}(z) U_{2}(z) .
$$

If $q \geq 3$, iterating the same procedure successively, we obtain that

$$
F(z)=F_{q}(z) V_{q}(z) P^{q}(z) U_{q}(z),
$$

where $V_{q}(z), U_{q}(z)$ are unimodular. Note that $\operatorname{det} F(z)=\left(z_{1}-f_{1}\left(z_{2}, \ldots, z_{n}\right)\right)^{q}\left(z_{2}-f_{2}\left(z_{3}, \ldots, z_{n}\right)\right)^{q}$, then $\operatorname{det} F_{q}(z)=c, c \in K$; that is, $F_{q}(z)$ is a unimodular matrix. So $F(z)$ is equivalent to its Smith form $P^{q}(z)$.

Necessity. It is straightforward that all the $(l-1) \times(l-1)$ minors of $P^{q}(z)$ generate $K[z]$. According to Lemma 3, all the $(l-1) \times(l-1)$ minors of $F(z)$ also generate $K[z]$.

Next we investigate Problem 1 for the case of $F(z) \in K^{l \times l}[z]$ and det $F(z)=\left(z_{1}-f_{1}\left(z_{2}, \ldots, z_{n}\right)\right)^{q_{1}}$ $\left(z_{2}-f_{2}\left(z_{3}, \ldots, z_{n}\right)\right)^{q_{2}}$, where $q_{1}, q_{2}$ are nonnegative integers.
Theorem 3. Let $F(z) \in K^{l \times l}[z]$ with $\operatorname{det} F(z)=\left(z_{1}-\right.$ $\left.f_{1}\left(z_{2}, \ldots, z_{n}\right)\right)^{q_{1}}\left(z_{2}-f_{2}\left(z_{3}, \ldots, z_{n}\right)\right)^{q_{2}}$, where $q_{1}, q_{2}$ are nonnegative integers. If all the $(l-1) \times(l-1)$ minors of $F(z)$ generate $K[z]$, then $F(z)$ is equivalent to its Smith form

$$
Q(z)=\left(\begin{array}{cc}
I_{l-1} & 0_{l-1,1} \\
0_{1, l-1} & \left(z_{1}-f_{1}\left(z_{2}, \ldots, z_{n}\right)\right)^{q_{1}}\left(z_{2}-f_{2}\left(z_{3}, \ldots, z_{n}\right)\right)^{q_{2}}
\end{array}\right) .
$$

Proof. (1) If $q_{1}=q_{2}=0$, then $\operatorname{det} F(z)=1$, i.e., $F(z)$ is unimodular, so $F(z)$ is equivalent to $I_{l}$.

(2) If $q_{1}=0$ or $q_{2}=0$, we have that $\operatorname{det} F(z)=\left(z_{1}-\right.$ $\left.f_{1}\left(z_{2}, \ldots, z_{n}\right)\right)^{q_{1}}$ or $\left(z_{2}-f_{2}\left(z_{3}, \ldots, z_{n}\right)\right)^{q_{2}}$. Without loss of generality, we assume that $\operatorname{det} F(z)=\left(z_{1}-f_{1}\left(z_{2}, \ldots, z_{n}\right)\right)^{q_{1}}$. Because $\operatorname{det} F\left(f_{1}\left(z_{2}, \ldots, z_{n}\right), z_{2}, \ldots, z_{n}\right)=0$, then the rank of $F\left(f_{1}\left(z_{2}, \ldots, z_{n}\right), z_{2}, \ldots, z_{n}\right) \leq l-1$. Since all the $(l-1) \times$ $(l-1)$ minors of $F(z)$ generate $K[z]$, by Lemma 5 , the ( $l-$ $1) \times(l-1)$ minors of $F\left(f_{1}\left(z_{2}, \ldots, z_{n}\right), z_{2}, \ldots, z_{n}\right)$ also generate $K[z]$; hence, $\operatorname{rank} F\left(f_{1}\left(z_{2}, \ldots, z_{n}\right), z_{2}, \ldots, z_{n}\right)=$ $l-1$ for every $\left(z_{2}, z_{3}, \ldots, z_{n}\right) \in \bar{K}^{n-1}$. By Lemma 4 , we obtain a ZRP column vector $Y_{1}(z) \in K^{l \times 1}[z]$ such that

$$
F\left(f_{1}\left(z_{1}, \ldots, z_{n}\right), z_{2}, \ldots, z_{n}\right) Y_{1}(z) \equiv(0, \ldots, 0)^{T} .
$$

According to the Quillen-Suslin Theorem, an $l \times l$ unimodular matrix $U_{11}(z)\left(\operatorname{det} U_{11}(z)=1\right)$ can be constructed such that $Y_{1}(z)$ is its last column. Then the last column of $F\left(f_{1}\left(z_{1}, \ldots, z_{n}\right), z_{2}, \ldots, z_{n}\right) \cdot U_{11}(z)$ are zero polynomials. By Lemma 2 , the last column of $F(z) \cdot U_{11}(z)$ have the common divisor $z_{1}-f_{1}\left(z_{2}, \ldots, z_{n}\right)$, i.e.,

$$
F(z) \cdot U_{11}(z)=F_{1}(z) \cdot\left(\begin{array}{cc}
I_{l-1} & 0_{l-1,1} \\
0_{1, l-1} & z_{1}-f_{1}\left(z_{2}, \ldots, z_{n}\right)
\end{array}\right),
$$

for some $F_{1}(z) \in K^{l \times l}[z]$. Let $U_{1}(z)=U_{11}^{-1}(z)$, then we obtain that

$$
\begin{aligned}
F(z) & =F_{1}(z) P_{1}(z) U_{1}(z), \\
\operatorname{det} F_{1}(z) & =\left(z_{1}-f_{1}\left(z_{2}, \ldots, z_{n}\right)\right)^{q_{1}-1} .
\end{aligned}
$$

By Lemma 3 , all the $(l-1) \times(l-1)$ minors of $F_{1}(z)$ also generate the unit ideal $K[z]$. If $q_{1} \geq 2$, iterating the preceding process, we obtain that

$$
F_{1}(z)=F_{2}(z) P_{1}(z) U_{2}(z) .
$$

Furthermore,

$$
F(z)=F_{2}(z) P_{1}(z) U_{2}(z) P_{1}(z) U_{1}(z) .
$$

By Lemma 3 , the $(l-1) \times(l-1)$ minors of $P_{1}(z) U_{2}(z) P_{1}(z)$ generate the ideal $K[z]$, according to Lemma 8, there exist unimodular matrices $N_{1}(z), V_{1}(z)$ such that $P_{1}(z) U_{2}(z) P_{1}(z)=N_{1}(z) P_{1}^{2}(z) V_{1}(z)$. Then

$$
F(z)=F_{2}(z) N_{1}(z) P_{1}^{2}(z) V_{1}(z) U_{1}(z) .
$$

Furthermore, $\quad \operatorname{det} F_{2}(z) N_{1}(z)=\left(z_{1}-f_{1}\left(z_{2}, \ldots\right.\right.$, $\left.\left.z_{n}\right)\right)^{q_{1}-2}$, combined with Lemma 3 , the $(l-1) \times(l-1)$ minors of $F_{2}(z) N_{1}(z)$ also generate $K[z]$. 
If $q_{1} \geq 3$, iterating the same procedure successively, we obtain that

$$
F(z)=F_{q_{1}}(z) N_{q_{1}-1}(z) P_{1}^{q_{1}}(z) V_{q_{1}-1}(z), \ldots, V_{1}(z) U_{1}(z),
$$

with $\operatorname{det} F_{q_{1}}(z) N_{q_{1}-1}(z)=1$. Thus, $F(z)$ is equivalent to its Smith form $P_{1}^{q_{1}}(z)=Q(z)$.

(3) If $q_{1}, q_{2}$ are positive integers, then $q_{1} \geq q_{2}$ or $q_{2} \geq q_{1}$. Without loss of generality, we assume that $q_{1} \geq q_{2}$. By Theorem 2, combined with the conclusion of the case (2) above, we have that $F(z)$ is equivalent to the matrix
$P^{q_{2}}(z) V(z) P_{1}^{q_{1}-q_{2}}(z)$. In the following, we prove that $P^{q_{2}}(z) V(z) P_{1}^{q_{1}-q_{2}}(z)$ is equivalent to the matrix $Q(z)$

Let

$$
V(z)=\left(\begin{array}{ll}
V_{11}(z) & V_{12}(z) \\
V_{21}(z) & V_{22}(z)
\end{array}\right)
$$

where $\quad V_{11}(z) \in K^{(l-1) \times(l-1)}[z], \quad V_{12}(z) \in K^{(l-1) \times 1}[z]$, $V_{21}(z) \in K^{1 \times(l-1)}[z]$, and $V_{22}(z) \in K^{1 \times 1}[z]$.

Then

$$
P^{q_{2}}(z) V(z) P_{1}^{q_{1}-q_{2}}(z)=\left(\begin{array}{cc}
V_{11}(z) & V_{12}(z) p_{1}^{q_{1}-q_{2}}(z) \\
V_{21}(z) p_{1}^{q_{2}}(z) p_{2}^{q_{2}}(z) & V_{22}(z) p_{1}^{q_{1}}(z) p_{2}^{q_{2}}(z)
\end{array}\right)
$$

where $\quad p_{1}(z)=z_{1}-f_{1}\left(z_{2}, \ldots, z_{n}\right), \quad p_{2}(z)=$ $z_{2}-f_{2}\left(z_{3}, \ldots, z_{n}\right)$.

In fact, we can prove that $\left(V_{11}(z) V_{12}(z) p_{1}^{q_{1}-q_{2}}(z)\right)$ is a ZLP matrix. Note that $V(z)$ is unimodular, then $\left(V_{11}(z) V_{12}(z)\right)$ is a ZLP matrix. Let $c_{1}(z), c_{2}(z), \ldots$, $c_{l}(z)$ denote all the $(l-1) \times(l-1)$ minors of $\left(V_{11}(z) V_{12}(z)\right)$. Then all the $(l-1) \times(l-1)$ minors of $\left(V_{11}(z) V_{12}(z) p_{1}^{q_{1}-q_{2}}(z)\right)$ are $c_{1}(z), c_{2}(z) p_{1}^{q_{1}-q_{2}}(z), \ldots$, $c_{l}(z) p_{1}^{q_{1}-q_{2}}(z)$. We can prove that $c_{1}(z), c_{2}(z) p_{1}^{q_{1}-q_{2}}(z), \ldots$, $c_{l}(z) p_{1}^{q_{1}-q_{2}}(z)$ have no common zeros. Suppose that $c_{1}(z), c_{2}(z) p_{1}^{q_{1}-q_{2}}(z), \ldots, c_{l}(z) p_{1}^{q_{1}-q_{2}}(z)$ have a common zero $\alpha_{0}$, note that $c_{1}(z), c_{2}(z), \ldots, c_{l}(z)$ have no common zeros, so $c_{1}(z)$ and $p_{1}(z)$ have the common zero $\alpha_{0}$. Moreover, the elements of the last row of matrix $P^{q_{2}}(z) V(z) P_{1}^{q_{1}-q_{2}}(z)$ have the common factor $p_{1}(z)$, since an $(l-1) \times(l-1)$ minors of $P^{q_{2}}(z) V(z) P_{1}^{q_{1}-q_{2}}(z)$ is just $c_{1}(z)$ or includes the factor $p_{1}(z)$, so the $(l-1) \times(l-1)$ minors of $P^{q_{2}}(z) V(z) P_{1}^{q_{1}-q_{2}}(z)$ have the common zero $\alpha_{0}$, this is a contradiction. Thus, all the $(l-1) \times(l-1)$ minors of $\left(V_{11}(z) V_{12}(z) p_{1}^{q_{1}-q_{2}}(z)\right)$ have no common zeros, and it is a ZLP matrix. By the Quillen-Suslin theorem, there exists an $l \times l$ unimodular matrix $N(z)$ such that

$$
\left(V_{11}(z) V_{12}(z) p_{1}^{q_{1}-q_{2}}(z)\right) N(z)=\left(\begin{array}{lll}
I_{l-1}(z) & 0_{l-1,1}
\end{array}\right) .
$$

Then,

$$
P^{q_{2}}(z) V(z) P_{1}^{q_{1}-q_{2}}(z) N(z)=\left(\begin{array}{cc}
I_{l-1}(z) & 0_{l-1,1} \\
H_{1}(z) & H_{2}(z)
\end{array}\right),
$$

for some $H_{1}(z), H_{2}(z) \in K[z]$. There exists a unimodular matrix $N_{1}(z)$ such that

$$
N_{1}(z) P^{q_{2}}(z) V(z) P_{1}^{q_{1}-q_{2}}(z) N(z)=\left(\begin{array}{cc}
I_{l-1}(z) & 0_{l-1,1} \\
0_{1, l-1} & H_{2}(z)
\end{array}\right) .
$$

Note that $N(z), N_{1}(z)$ are unimodular, then $H_{2}(z)=$ $u \cdot \operatorname{det} P^{q_{2}}(z) V(z) P_{1}^{q_{1}-q_{2}}(z)=u \iota \cdot p_{1}^{q_{1}}(z) p_{2}^{q_{2}}(z), 0 \neq u \in K$, $u \prime=u \cdot c$; thus, $P^{q_{2}}(z) V(z) P_{1}^{q_{1}-q_{2}}(z)$ is equivalent to $Q(z)$. Note that $F(z)$ is equivalent to the matrix
$P^{q_{2}}(z) V(z) P_{1}^{q_{1}-q_{2}}(z)$, combined with the definition of the Smith form of a matrix, we obtain that $F(z)$ is equivalent to its Smith form $Q(z)$.

Theorem 3 provides a positive answer to Problem 1 for the case of $F(z) \in K^{l \times l}[z]$ and $\operatorname{det} F(z)=\left(z_{1}-f_{1}\left(z_{2}, \ldots\right.\right.$, $\left.\left.z_{n}\right)\right)^{q_{1}}\left(z_{2}-f_{2}\left(z_{3}, \ldots, z_{n}\right)\right)^{q_{2}}$. It also gives a sufficient condition to check this kind of matrices are equivalent to their Smith forms; in fact, this condition is also a necessary condition.

Theorem 4. Let $F(z) \in K^{l \times l}[z]$ with $\operatorname{det} F(z)=\left(z_{1}-\right.$ $\left.f_{1}\left(z_{2}, \ldots, z_{n}\right)\right)^{q_{1}}\left(z_{2}-f_{2}\left(z_{3}, \ldots, z_{n}\right)\right)^{q_{2}}$, where $q_{1}, q_{2}$ are non-negative integers, then $F(z)$ is equivalent to its Smith form

$$
Q(z)=\left(\begin{array}{cc}
I_{l-1} & 0_{l-1,1} \\
0_{1, l-1} & \left(z_{1}-f_{1}\left(z_{2}, \ldots, z_{n}\right)\right)^{q_{1}}\left(z_{2}-f_{2}\left(z_{3}, \ldots, z_{n}\right)\right)^{q_{2}}
\end{array}\right),
$$

if and only if all the $(l-1) \times(l-1)$ minors of $F(z)$ generate $K[z]$.

Proof. Sufficiency. From Theorem 3, it is straightforward.

Necessity. By computing, we can easily obtain that all the $(l-1) \times(l-1)$ minors of $Q(z)$ generate $K[z]$. By Lemma 3, all the $(l-1) \times(l-1)$ minors of $F(z)$ generate $K[z]$.

Remark 1. In the theorem above, $K$ is an arbitrary field. When $K=\mathbb{R}$ is the real field and $q_{2}=0$, Theorem 4 is same as Theorem $2.5 \mathrm{in} \mathrm{Li} \mathrm{et} \mathrm{al.} \mathrm{[17].} \mathrm{Furthermore,} \mathrm{if} q_{1}=1$ and $q_{2}=0$, Theorem 4 is just Proposition 4 in Lin et al. [12]. So Theorem 4 extends the above two results.

In the following, we will investigate the Smith forms of some rectangular polynomial matrices and consider Problem 2. Let $d(z)$ denote the greatest common divisor (g.c.d) of the $l \times l$ minors of the matrix $F(z), d_{l-1}(F(z))$ denote the g.c.d of all the $(l-1) \times(l-1)$ minors of $F(z)$ and denote 


$$
B(z)=\left(\begin{array}{cc}
I_{l-1} & 0_{l-1,1} \\
0_{1, l-1} & d(z)
\end{array}\right) .
$$

Theorem 5. Let $F(z) \in K^{l \times m}[z](l \leq m)$ be of full row rank and suppose $d(z)=\left(z_{1}-f_{1}\left(z_{2}, \ldots, z_{n}\right)\right)^{q_{1}}\left(z_{2}-f_{2} \quad\left(z_{3}, \ldots, z_{n}\right)\right)^{q_{2}}$, where $q_{1}, q_{2}$ are nonnegative integers. Then $F(z)$ is equivalent to its Smith form

$$
Q(z)=\left(B(z) 0_{l \times(m-l)}\right),
$$

if and only if all the $(l-1) \times(l-1)$ minors of $F(z)$ generate $K[z]$.

Proof. Sufficiency. According to Theorem 3.3 in [21], there exists $\quad G(z) \in K^{l \times l}[z], \quad F_{0}(z) \in K^{l \times m}[z] \quad$ such that $F(z)=G(z) \cdot F_{0}(z)$, where $\operatorname{det} G(z)=d(z)$, and $F_{0}(z)$ is a ZLP matrix. Combined with Theorem 5, we can obtain two unimodular matrices $V_{1}(z), V_{2}(z) \in K^{l \times l}[z]$ such that $G(z)=V_{1}(z) \cdot B(z) \cdot V_{2}(z)$. Then

$$
F(z)=V_{1}(z) \cdot B(z) \cdot V_{2}(z) \cdot F_{0}(z) .
$$

It is obviously that $V_{2}(z) \cdot F_{0}(z)$ is also ZLP. According to the Quillen-Suslin Theorem, we can construct an $m \times m$ unimodular matrix $U(z)$ such that $V_{2}(z) \cdot F_{0}(z)$ is its first $l$ rows. Hence

$$
F(z)=V_{1}(z) \cdot\left(\begin{array}{ll}
B(z) & 0_{l, m-l}
\end{array}\right) \cdot U(z)=V_{1}(z) \cdot Q(z) \cdot U(z),
$$

and $d_{l-1}(Q(z))=1$. Since $Q(z)=V_{1}^{-1}(z) \cdot F(z) \cdot U^{-1}(z)$, combined with Lemma 3 , we have that $d_{l-1}(F(z))=1$, then the Smith form of $F(z)$ is $Q(z)$.

Necessity. If $F(z)$ is equivalent to its Smith form $Q(z)=\left(B(z) 0_{l,(m-l)}\right)$, we see that all the $(l-1) \times(l-1)$ minors of $Q(z)$ generate $K[z]$. Using Lemma 3, we obtain that all the $(l-1) \times(l-1)$ minors of $F(z)$ generate $K[z]$.

Remark 2. Let $f_{1}(z), f_{2}(z), \ldots, f_{t}(z) \in K[z]$ be nonzero polynomials, a necessary and sufficient condition for $G=$ $\left\{f_{1}(z), f_{2}(z), \ldots, f_{t}(z)\right\}$ generates that $K[z]$ is the reduced Gröbner basis of the ideal generated by $G$ includes a unit in the field $K$. According to Theorems 4 and 5, we can check whether an $n \mathrm{D}$ polynomial matrix $F(z)$ is equivalent to its Smith form by using the existing Gröbner basis algorithms to the ideal generated by the lower minors of $F(z)$. Thus, the conditions of Theorems 4 and 5 can be verified easily.

\section{Example and Algorithm}

In this section, we first present a $2 \mathrm{D}$ example to illustrate our result and explain how to obtain the unimodular matrices associated with the equivalence of system matrices in the method. Then we give an algorithm to deal with the equivalence of the kind of matrices we discussed to their Smith forms.

In many areas of engineering such as Circuits and Signals, the general 2D systems can be defined in terms of the generalized Rosenbrock system [8] as

$$
\begin{aligned}
F\left(z_{1}, z_{2}\right) x & =U\left(z_{1}, z_{2}\right) u, \\
y & =V\left(z_{1}, z_{2}\right) x+W\left(z_{1}, z_{2}\right) u,
\end{aligned}
$$

where $x \in K^{n}$ is the state vector, $u \in K^{l}$ is the input vector, $y \in K^{m}$ is the output vector, $F\left(z_{1}, z_{2}\right) \in K^{n \times n}\left[z_{1}, z_{2}\right]$, $U\left(z_{1}, z_{2}\right) \in K^{n \times l}\left[z_{1}, z_{2}\right], \quad V\left(z_{1}, z_{2}\right) \in K^{m \times n}\left[z_{1}, z_{2}\right]$, $W\left(z_{1}, z_{2}\right) \in K^{m \times l}\left[z_{1}, z_{2}\right]$ are polynomial matrices, $K$ is a field. The operators $z_{1}$ and $z_{2}$ may have various meanings depending on the type of system. For example, in delaydifferential systems, $z_{1}$ represents a differential operator and $z_{2}$ a delay-operator. For $2 \mathrm{D}$ discrete systems, $z_{1}$ and $z_{2}$ represent horizontal and vertical shift operators, respectively. This system gives rise to the system matrix in the general form:

$$
T\left(z_{1}, z_{2}\right)=\left(\begin{array}{cc}
F\left(z_{1}, z_{2}\right) & U\left(z_{1}, z_{2}\right) \\
-V\left(z_{1}, z_{2}\right) & W\left(z_{1}, z_{2}\right)
\end{array}\right) .
$$

Example 1. Consider a system matrix

$$
\begin{aligned}
T\left(z_{1}, z_{2}\right) & =\left(\begin{array}{cc}
F\left(z_{1}, z_{2}\right) & U\left(z_{1}, z_{2}\right) \\
-V\left(z_{1}, z_{2}\right) & W\left(z_{1}, z_{2}\right)
\end{array}\right) \\
& =\left(\begin{array}{cccc}
z_{2}^{3} & -6 z_{2}^{2} z_{1}^{2}-8 z_{2}^{2} z_{1}-z_{2}^{2}+4 z_{2} z_{1}^{2}+2 z_{2} z_{1}-z_{1}^{2} & 0 & z_{1} \\
z_{1}^{2} & -4-8 z_{1}+z_{2} z_{1}-2 z_{2}^{2}+z_{2}^{3} & z_{1}+1 & z_{2} \\
z_{1}-1 & 2 z_{2}^{2}-z_{1} z_{2}-6 z_{2}+4 z_{1} & 1 & 0 \\
z_{1} & z_{2} & 0 & 0
\end{array}\right),
\end{aligned}
$$

where 


$$
\begin{aligned}
F\left(z_{1}, z_{2}\right) & =\left(\begin{array}{ccc}
z_{2}^{3} & -6 z_{2}^{2} z_{1}^{2}-8 z_{2}^{2} z_{1}-z_{2}^{2}+4 z_{2} z_{1}^{2}+2 z_{2} z_{1}-z_{1}^{2} & 0 \\
z_{1}^{2} & -4-8 z_{1}+z_{2} z_{1}-2 z_{2}^{2}+z_{2}^{3} & z_{1}+1 \\
z_{1}-1 & 2 z_{2}^{2}-z_{1} z_{2}-6 z_{2}+4 z_{1} & 1
\end{array}\right), \\
U\left(z_{1}, z_{2}\right) & =\left(z_{1} z_{2} 0\right)^{T}, \\
V\left(z_{1}, z_{2}\right) & =\left(-z_{1}-z_{2} 0\right), \\
W\left(z_{1}, z_{2}\right) & =(0) .
\end{aligned}
$$

By computing, $\operatorname{det} F(z)=\left(z_{1}-z_{2}\right)^{2}\left(z_{2}-1\right)^{4}$, the $2 \times 2$ minors of $F(z)$ is as follows: $c_{1}(z)=z_{2}^{6}-2 z_{2}^{5}+z_{2}^{4} z_{1}-$ $8 z_{2}^{3} z_{1}-4 z_{2}^{3}+6 z_{2}^{2} z_{1}^{4}+8 z_{2}^{2} z_{1}^{3}+z_{2}^{2} z_{1}^{2}-4 z_{2} z_{1}^{4}-2 z_{2} z_{1}^{3}+z_{1}^{4}, c_{2}(z)=$ $z_{2}^{3}\left(z_{1}+1\right), \quad c_{3}(z)=-\left(z_{1}+1\right)\left(6 z_{2}^{2} z_{1}^{2}+8 z_{2}^{2} z_{1}+z_{2}^{2}-4 z_{2} z_{1}^{2}-\right.$ $\left.2 z_{2} z_{1}+z_{1}^{2}\right), \quad c_{4}(z)=2 z_{2}^{5}-z_{2}^{4} z_{1}-6 z_{2}^{4}+4 z_{2}^{3} z_{1}+6 z_{2}^{2} z_{1}^{3}+2 z_{2}^{2} z_{1}^{2}-$ $7 z_{2}^{2} z_{1}-z_{2}^{2}-4 z_{2} z_{1}^{3}+2 z_{2} z_{1}^{2}+2 z_{2} z_{1}+z_{1}^{3}-z_{1}^{2}, c_{5}(z)=z_{1}^{3}, c_{6}(z)=$ $-6 z_{2}^{2} z_{1}^{2}-8 z_{2}^{2} z_{1}-z_{2}^{2}+4 z_{2} z_{1}^{2}+2 z_{2} z_{1}-z_{1}^{2}, \quad c_{7}(z)=-z_{2}^{3} z_{1}+z_{2}^{3}+$ $2 z_{2}^{2} z_{1}^{2}+2 z_{2}^{2} z_{1}-2 z_{2}^{2}-z_{2} z_{1}^{3}-7 z_{2} z_{1}^{2}+z_{2} z_{1}+4 z_{1}^{3}+8 z_{1}^{2}-4 z_{1}-4$, $c_{8}(z)=1, \quad c_{9}(z)=z_{2}^{3}-2 z_{2}^{2} z_{1}-4 z_{2}^{2}+z_{2} z_{1}^{2}+8 z_{2} z_{1}+6 z_{2}-4 z_{1}^{2}-$ $12 z_{1}-4$.

The reduced Gröbner basis of the ideal generated by $c_{1}(z), \ldots, c_{9}(z)$ is $\{1\}$. So the $2 \times 2$ minors of $F\left(z_{1}, z_{2}\right)$ generate unit ideal $K\left[z_{1}, z_{2}\right]$, by Theorem $4, F\left(z_{1}, z_{2}\right)$ is equivalent to its Smith form. Consider

$$
F\left(z_{1}, 1\right)=\left(\begin{array}{ccc}
1 & -3 z_{1}^{2}-6 z_{1}-1 & 0 \\
z_{1}^{2} & -7 z_{1}-5 & z_{1}+1 \\
z_{1}-1 & 2 z_{1}-4 & 1
\end{array}\right) .
$$

Solving the equations, $F\left(z_{1}, 1\right) Y_{1}=0$, where $Y_{1} \in K^{3 \times 1}\left[z_{1}\right]$, we

obtain $Y_{1}^{T}=\left(3 z_{1}^{2}+6 z_{1}+1,1,-3 z_{1}^{3}-3 z_{1}^{2}+2 z_{1}+5\right)$, where $Y_{1}^{T}$ is the transpose of $Y_{1}$. It then follows that

$$
F\left(z_{1}, 1\right)\left(\begin{array}{c}
3 z_{1}^{2}+6 z_{1}+1 \\
1 \\
-3 z_{1}^{3}-3 z_{1}^{2}+2 z_{1}+5
\end{array}\right)=\left(\begin{array}{l}
0 \\
0 \\
0
\end{array}\right)
$$

We complete $\left(3 z_{1}^{2}+6 z_{1}+1,1,-3 z_{1}^{3}-3 z_{1}^{2}+2 z_{1}+5\right)^{T}$ into a unimodular matrix $U_{41} \in K^{3 \times 3}\left[z_{1}\right]$ such that $F U_{41}=F_{1} P_{2}$, where

$$
\begin{aligned}
F_{1} & =\left(\begin{array}{ccc}
z_{2}^{3} & 0 & 3 z_{2}^{2} z_{1}^{2}+6 z_{2}^{2} z_{1}+z_{2}^{2}-3 z_{2} z_{1}^{2}-2 z_{2} z_{1}+z_{1}^{2} \\
z_{1}^{2} & -z_{1}-1 & z_{2}^{2}-z_{2}+z_{1}-1 \\
z_{1}-1 & -1 & 2 z_{2}-z_{1}-4
\end{array}\right) \\
P_{2} & =\left(\begin{array}{ccc}
1 & 0 & 0 \\
0 & 1 & 0 \\
0 & 0 & z_{2}-1
\end{array}\right) \\
U_{41} & =\left(\begin{array}{ccc}
1 & 0 & 3 z_{1}^{2}+6 z_{1}+1 \\
0 & 0 & 1 \\
0 & -1 & -3 z_{1}^{3}-3 z_{1}^{2}+2 z_{1}+5
\end{array}\right)
\end{aligned}
$$

We then have

$$
F=F_{1} P_{2} U_{4}
$$

where

$$
U_{4}=U_{41}^{-1}=\left(\begin{array}{ccc}
1 & -3 z_{1}^{2}-6 z_{1}-1 & 0 \\
0 & -3 z_{1}^{3}-3 z_{1}^{2}+2 z_{1}+5 & -1 \\
0 & 1 & 0
\end{array}\right)
$$

We consider $F_{1}\left(z_{1}, z_{2}\right)$ again

Repeating the procedure above for $F_{1}$, we have that

$$
F_{1}=F_{2} P_{2} U_{3} \text {, }
$$

where 


$$
\begin{aligned}
F_{2} & =\left(\begin{array}{ccc}
z_{2}^{3} & 0 & z_{2}^{2} z_{1}^{2}+4 z_{2}^{2} z_{1}+z_{2}^{2}-2 z_{2} z_{1}^{2}-2 z_{2} z_{1}+z_{1}^{2} \\
z_{1}^{2} & z_{1}+1 & -z_{2} \\
z_{1}-1 & 1 & -2
\end{array}\right), \\
U_{3} & =\left(\begin{array}{ccc}
1 & 0 & z_{1}^{2}+4 z_{1}+1 \\
0 & -1 & -z_{1}^{3}-3 z_{1}^{2}+2 z_{1}-1 \\
0 & 0 & -1
\end{array}\right) .
\end{aligned}
$$

Then,

$$
F=F_{1} P_{2} U_{4}=F_{2} P_{2} U_{3} P_{2} U_{4} .
$$

By Theorem 3.5 in [17], we have that $P_{2} U_{3} P_{2}$ is equivalent to $P_{2}^{2}$, and

$$
P_{2} U_{3} P_{2}=P_{2}^{2} M_{2},
$$

where

$$
M_{2}=\left(\begin{array}{ccc}
1 & 0 & \left(z_{2}-1\right)\left(z_{1}^{2}+4 z_{1}+1\right) \\
0 & -1 & -\left(z_{2}-1\right)\left(z_{1}^{3}+3 z_{1}^{2}-2 z_{1}+1\right) \\
0 & 0 & -1
\end{array}\right) .
$$

So,

$$
F=F_{1} P_{2} U_{4}=F_{2} P_{2} U_{3} P_{2} U_{4}=F_{2} P_{2}^{2} M_{2} U_{4} .
$$

Consider $F_{2}$, factor it like above, we obtain that

$$
F_{2}=F_{3} P_{2} U_{2} \text {, }
$$

where

$$
\begin{aligned}
F_{3} & =\left(\begin{array}{ccc}
z_{2}^{3} & 0 & 2 z_{2}^{2} z_{1}+z_{2}^{2}-z_{2} z_{1}^{2}-2 z_{2} z_{1}+z_{1}^{2} \\
z_{1}^{2} & -z_{1}-1 & 1 \\
z_{1}-1 & -1 & 0
\end{array}\right), \\
U_{2} & =\left(\begin{array}{ccc}
1 & 0 & 2 z_{1}+1 \\
0 & -1 & 2 z_{1}^{2}-z_{1}+1 \\
0 & 0 & -1
\end{array}\right) .
\end{aligned}
$$

Then,

$$
F=F_{2} P_{2}^{2} M_{2} U_{4}=F_{3} P_{2} U_{2} P_{2}^{2} M_{2} U_{4} .
$$

We consider $F_{3}$ again

$$
F_{3}\left(z_{2}, z_{2}\right)=\left(\begin{array}{ccc}
z_{2}^{3} & 0 & z_{2}^{3} \\
z_{2}^{2} & -z_{2}-1 & 1 \\
z_{2}-1 & -1 & 0
\end{array}\right) .
$$

Solving the equations, $F_{3}\left(z_{2}, z_{2}\right) Y_{2}=0$, where $Y_{2} \in K^{3 \times 1}\left[z_{2}\right]$, we obtain $Y_{2}^{T}=\left(1, z_{2}-1,-1\right)$, where $Y_{2}^{T}$ is the transpose of $Y_{2}$. It then follows that

$$
F_{3}\left(z_{2}, z_{2}\right)\left(\begin{array}{c}
1 \\
z_{2}-1 \\
-1
\end{array}\right)=\left(\begin{array}{l}
0 \\
0 \\
0
\end{array}\right)
$$

We complete $\left(1, z_{2}-1,-1\right)^{T}$ into a unimodular matrix $V_{21} \in K^{3 \times 3}\left[z_{2}\right]$ such that $F_{3} V_{21}=F_{4} P_{1}$, where

$$
\begin{aligned}
F_{4} & =\left(\begin{array}{ccc}
z_{2}^{3} & 0 & \left(z_{2}-1\right)\left(z_{1}-z_{2}\right) \\
z_{1}^{2} & z_{1}+1 & z_{1}+1 \\
z_{1}-1 & 1 & 1
\end{array}\right), \\
P_{1} & =\left(\begin{array}{ccc}
1 & 0 & 0 \\
0 & 1 & 0 \\
0 & 0 & z_{1}-z_{2}
\end{array}\right) \\
V_{21} & =\left(\begin{array}{ccc}
1 & 0 & 1 \\
0 & -1 & z_{2}-1 \\
0 & 0 & -1
\end{array}\right) .
\end{aligned}
$$

We obtain that

$$
F_{3}=F_{4} P_{1} V_{2} \text {, }
$$

where

$$
V_{2}=V_{21}^{-1}=\left(\begin{array}{ccc}
1 & 0 & 1 \\
0 & -1 & 1-z_{2} \\
0 & 0 & -1
\end{array}\right)
$$

Thus,

$$
F=F_{3} P_{2} U_{2} P_{2}^{2} M_{2} U_{4}=F_{4} P_{1} V_{2} P_{2} U_{2} P_{2}^{2} M_{2} U_{4} .
$$

Now we consider $P_{1} V_{2} P_{2}$. By Lemma 7, we have that $P_{1} V_{2} P_{2}$ is equivalent to $P$. And

$$
P_{1} V_{2} P_{2}=P M_{1} \text {, }
$$

where

$$
M_{1}=\left(\begin{array}{ccc}
1 & 0 & z_{2}-1 \\
0 & -1 & -\left(z_{2}-1\right)^{2} \\
0 & 0 & -1
\end{array}\right) .
$$

Thus,

$$
F=F_{4} P_{1} V_{2} P_{2} U_{2} P_{2}^{2} M_{2} U_{4}=F_{4} P M_{1} U_{2} P_{2}^{2} M_{2} U_{4} .
$$

Repeating the procedure above for $F_{4}$, we obtain that $F_{4}=F_{5} P_{2} U_{1}$. Furthermore, we consider $F_{5}$ and obtain $F_{5}=F_{6} P_{1}$. So

$$
\begin{aligned}
F & =F_{4} P M_{1} U_{2} P_{2}^{2} M_{2} U_{4}=F_{5} P_{2} U_{1} P M_{1} U_{2} P_{2}^{2} M_{2} U_{4} \\
& =F_{6} P_{1} P_{2} U_{1} P M_{1} U_{2} P_{2}^{2} M_{2} U_{4},
\end{aligned}
$$

where 


$$
\begin{aligned}
F_{5} & =\left(\begin{array}{ccc}
z_{2}^{3} & 0 & 1-z_{2} \\
z_{1}^{2} & -z_{1}-1 & 0 \\
z_{1}-1 & -1 & 0
\end{array}\right), \\
F_{6} & =\left(\begin{array}{ccc}
z_{2}^{3} & 0 & -1 \\
z_{1}^{2} & -z_{1}-1 & 0 \\
z_{1}-1 & -1 & 0
\end{array}\right), \\
U_{1} & =\left(\begin{array}{ccc}
1 & 0 & 0 \\
0 & -1 & -1 \\
0 & 0 & -1
\end{array}\right) .
\end{aligned}
$$

Setting

$$
P=P_{1} P_{2}\left(\begin{array}{ccc}
1 & 0 & 0 \\
0 & 1 & 0 \\
0 & 0 & \left(z_{1}-z_{2}\right)\left(z_{2}-1\right)
\end{array}\right),
$$

we obtain that

$$
F=F_{6} P_{1} P_{2} U_{1} P M_{1} U_{2} P_{2}^{2} M_{2} U_{4}=F_{6} P U_{1} P M_{1} U_{2} P_{2}^{2} M_{2} U_{4} \text {. }
$$

Let $L_{1}=M_{1} U_{2}, L_{2}=M_{2} U_{4}$, we have

$$
F=F_{6} P U_{1} P L_{1} P_{2}^{2} L_{2} \text {. }
$$

Now we consider the matrix $P U_{1} P$. From Theorem 2, we know that $P U_{1} P$ is equivalent to $P^{2}$. And

$$
P U_{1} P=P^{2} M_{3} \text {, }
$$

where

$$
M_{3}=\left(\begin{array}{ccc}
1 & 0 & 0 \\
0 & -1 & \left(z_{2}-z_{1}\right)\left(z_{2}-1\right) \\
0 & 0 & -1
\end{array}\right)
$$

Then we have

$$
F=F_{6} P^{2} M_{3} L_{1} P_{2}^{2} L_{2} .
$$

Let $L_{3}=M_{3} L_{1}$, now consider the matrix $P^{2} L_{3} P_{2}^{2}$. By Theorem 3, we have that $P^{2} L_{3} P_{2}^{2}$ is equivalent to $B$, where

$$
\begin{aligned}
B & =\left(\begin{array}{ccc}
1 & 0 & 0 \\
0 & 1 & 0 \\
0 & 0 & \left(z_{1}-z_{2}\right)^{2}\left(z_{2}-1\right)^{4}
\end{array}\right), \\
P^{2} L_{3} P_{2}^{2} & =B L_{4},
\end{aligned}
$$

where

$$
L_{4}=\left(\begin{array}{ccc}
1 & 0 & \left(z_{2}-1\right)^{2}\left(2 z_{1}-z_{2}+2\right) \\
0 & -1 & \left(z_{2}-1\right)^{2}\left(2 z_{1}^{2}-z_{2} z_{1}+z_{2}\right) \\
0 & 0 & -1
\end{array}\right) .
$$

Now

$$
F=F_{6} B L_{4} L_{2} .
$$

Let $L_{5}=L_{4} L_{2}$, then

$$
L_{5}=\left(\begin{array}{ccc}
1 & z_{2}^{3}-2 z_{2}^{2} z_{1}-4 z_{2}^{2}+z_{2} z_{1}^{2}+8 z_{2} z_{1}+6 z_{2}-4 z_{1}^{2}-12 z_{1}-4 & 0 \\
0 & z_{2}^{3} z_{1}-z_{2}^{3}-2 z_{2}^{2} z_{1}^{2}-2 z_{2}^{2} z_{1}+2 z_{2}^{2}+z_{2} z_{1}^{3}+7 z_{2} z_{1}^{2}-z_{2} z_{1}+4 z_{1}^{3}-8 z_{1}^{2}+4 z_{1}+4 & -1 \\
0 & 1 & 0
\end{array}\right),
$$

we have

$$
F=F_{6} B L_{5} .
$$

By computing, $\operatorname{det} F_{6}=\operatorname{det} F_{5}=1$.

Thus $F$ is equivalent to the matrix

$$
B=\left(\begin{array}{ccc}
1 & 0 & 0 \\
0 & 1 & 0 \\
0 & 0 & \left(z_{1}-z_{2}\right)^{2}\left(z_{2}-1\right)^{4}
\end{array}\right)
$$

Now we can find $X=0_{1 \times 3}, Y=0_{3 \times 1}$, such that

$$
\left(\begin{array}{cc}
F_{6}^{-1} & 0_{3 \times 1} \\
X & 1
\end{array}\right)\left(\begin{array}{cc}
F & U \\
-V & W
\end{array}\right)=\left(\begin{array}{cc}
B & U_{1} \\
-V_{1} & W_{1}
\end{array}\right)\left(\begin{array}{cc}
L_{5} & Y \\
0_{1 \times 3} & 1
\end{array}\right),
$$

where $\quad U_{1}=F_{6} U=\left(z_{2} z_{2} z_{1}-z_{2} z_{2}^{4}-z_{1}\right)^{T}, \quad V_{1}=V L_{5}^{-1}=$ $\left(-z_{1} 0-a_{1}\right), \quad a_{1}=-z_{1} z_{2}^{3}+2 z_{1}^{2} z_{2}^{2}+4 z_{1} z_{2}^{2}-z_{2} z_{1}^{3}-8 z_{2} z_{1}^{2}-$ $6 z_{2} z_{1}+z_{2}+4 z_{1}^{3}+12 z_{1}^{2}+4 z_{1}, W_{1}=0$.

Thus, the system

$$
\left(\begin{array}{cc}
F\left(z_{1}, z_{2}\right) & U\left(z_{1}, z_{2}\right) \\
-V\left(z_{1}, z_{2}\right) & W\left(z_{1}, z_{2}\right)
\end{array}\right)
$$

is equivalent to the system

$$
\left(\begin{array}{cc}
B\left(z_{1}, z_{2}\right) & U_{1}\left(z_{1}, z_{2}\right) \\
-V_{1}\left(z_{1}, z_{2}\right) & W_{1}\left(z_{1}, z_{2}\right)
\end{array}\right),
$$

which is simpler.

With the help of Example 1 and Theorem 3, we now can get the Algorithm 1.

The program of the function SyzygyModule which we use in algorithm can be found in https://faculty.math. illinois.edu/Macaulay2/doc/Macaulay2-1.15/share/doc/ Macaulay2/MCMApproximations/html/_syzygy_Module. html.

Another function CompleteMatrix is available in http:// wwwb.math.rwth-aachen.de/QuillenSuslin. Moreover, for a new algorithm for CompleteMatrix, see the package MatrixFactorization in http://www.mmrc.iss.ac.cn/dwang/ software.html, which contained a ZLP algorithm. This 
(i) Step 1. Declare the ring $K[z]=K\left[z_{1}, \ldots, z_{n}\right]$ over which the matrix is defined by declaring the indeterminates and the field of coefficients. Factor the determinant of $F$. Check that the determinant of $F$ is the form $\left(z_{j}-f_{j}\left(z_{2}, \ldots, z_{n}\right)\right)^{q_{j}}\left(z_{k}-f_{k}\left(z_{3}, \ldots, z_{n}\right)\right)^{q_{k}}$. If yes, set $q_{1}=\max \left\{q_{j}, q_{k}\right\}$, the polynomial it corresponds is $z_{1}-f_{1}$, $q_{2}=\min \left\{q_{j}, q_{k}\right\}$, the polynomial it corresponds is $z_{2}-f_{2}$, go to Step 2. Otherwise, return this method is not fit for $F$.

(ii) Step 2. Compute the reduced Gröbner basis $G$ of ideal generated by the lower minors of $F$. If $G=\{1\}$, go to Step 3; otherwise, return this method is not fit for $F$.

(iii) Step 3. Set $F_{0}=F, P_{1}=\operatorname{diag}\left(1, \ldots, 1, z_{1}-f_{1}\right), P_{2}=\operatorname{diag}\left(1, \ldots, 1, z_{2}-f_{2}\right), P=P_{1} P_{2}, i=0$ and $t=q_{1}-q_{2}$.

(iv) Step 4. Substitute $z_{1}=f_{1}$ in $F_{i}$ to obtain $\overline{F_{i}}$. Compute a ZRP vector $Y_{i} \in K\left[z_{2}, \ldots, z_{n}\right]$ such that $\overline{F_{i}} Y_{i}=0$ by using the function SyzygyModule. Then compute a unimodular matrix $U$ with $Y_{i}$ is its last column by using the function CompleteMatrix. Compute $F_{i+1}$ such that $F_{i} U=F_{i+1} P_{1}$. Compute $U^{-1}$ and set $U_{i+1}=U^{-1}$. Compute $P_{1}^{i} U_{i+1} P_{1}=N_{i} P_{1}^{i+1} V_{i}$ and obtain $F=F_{i+1} N_{i} P_{1}^{i+1} V_{i}$, store $F_{i+1} . L_{3}=F_{q_{1}} L_{1}, i=i+1$.

(v) Step 5. When $i \leq t$, go to Step 4. When $t<i<q_{1}$, do Step 6; otherwise, compute $P^{q_{2}} N_{q_{1}} P_{1}^{t}=L_{1} P^{q_{2}} P_{1}^{t} L_{2}$, let return $F=L_{3} P^{q_{2}} P_{1}^{t} L_{2}$.

(vi) Step 6. Substitute $z_{1}=f_{1}$ in $F_{i}$ to obtain $\overline{F_{i}}$, do procedure similar to the step 4 . And obtain a ZRP vector $Y_{i}$ such that $\overline{F_{i}} Y_{i}=0$ and a unimodular matrix $U$ with $Y_{i}$ is its last column. Then compute $F_{i+1}$ such that $F_{i} U=F_{i+1} P_{1}$, and compute $U^{-1}$ and set $U_{i+1}=U^{-1}$. Set $F_{i}=F_{i+1}$, substitute $z_{2}=f_{2}$ in $F_{i}$ to obtain $\overline{F_{i}}$. Compute a ZRP vector $X \in K\left[z_{1}, z_{3}, \ldots, z_{n}\right]$ such that $\overline{F_{i}} X=0$ by using the function SyzygyModule. Then compute a unimodular matrix $V$ with $X$ is its last column by using the function CompleteMatrix. Compute $V_{i-t}=V^{-1}$ and $F_{i+1}$ such that $F_{i} V_{i-t}=F_{i+1} P_{2}$. Compute $P_{1} U_{i+1} P_{2}=N_{i} P M_{i}$ and obtain $F=F_{i+1} P^{i-t} N_{i} P_{1}^{t} V_{i}$, where $V_{i}=M_{i} V_{i-t}$. Store $F_{i+1}, i=i+1$. Go to Step 5 .

Algorithm 1: A matrix equivalence (ME) algorithm.

algorithm can obtain a unimodular matrix whose inverse is the complete matrix of a given ZLP matrix.

\section{Conclusions}

In this paper, we have investigated the equivalence problem of several kinds of $n \mathrm{D}$ polynomials matrices over an arbitrary field, and have presented some interesting results. We have obtained some criteria for these matrices to equivalent to their Smith forms respectively. These criteria are easily checked by the existing Gröbner basis algorithm for the ideal generated by the minors of lower order of a given matrix. We also give an example to illustrate our method. All of these could provide useful information for engineers to reduce $n \mathrm{D}$ systems.

\section{Data Availability}

The data used to support the findings of this study are included within the article.

\section{Conflicts of Interest}

The authors declare that there are no conflicts of interest regarding the publication of this paper.

\section{Acknowledgments}

The authors are grateful to Professor Sang-Bing Tsai and the anonymous referees for their numerous instructive comments, which have significantly improved the presentation of the paper. This research is supported by the National Natural Science Foundation of China (11871207) and the Graduate Research and Innovation Project of Hunan Province in China (CX2018B674).

\section{References}

[1] N. Bose, Applied Multidimensional Systems Theory, Van Nostrand Reinhold, New York, NY, USA, 1982.

[2] N. Bose, B. Buchberger, and J. Guiver, Multidimensional Systems Theory and Applications, Kluwer, Dordrecht, Netherlands, 2003.

[3] J. Liu and M. Wang, "New results on multivariate polynomial matrix factorizations," Linear Algebra and Its Applications, vol. 438, no. 1, pp. 87-95, 2013.

[4] M. S. Boudellioua, K. Galkowski, and E. Rogers, "Characterization of a class of spatially interconnected systems (ladder circuits) using two-dimensional systems theory," Multidimensional Systems and Signal Processing, vol. 30, no. 4, pp. 2185-2197, 2019.

[5] S. Kung, B. Levy, M. Morf, and T. Kailath, "New results in 2-D systems theory: part II," Proceedings of the IEEE, vol. 65, pp. 945-961, 1977.

[6] E. Lee and S. Zak, "Smith form over $R\left[z_{1}, z_{2}\right]$," IEEE Transactions on Automatic Control, vol. 28, no. 1, pp. 115-118, 1983.

[7] H. H. Rosenbrock, State Space and Multivariable Theory, Nelson-Wiley, New York, NY, USA, 1970.

[8] D. Vafiadis and N. Karcanias, "Unimodular equivalence and similarity for linear systems," International Journal of Control, vol. 92, no. 9, pp. 2091-2098, 2018.

[9] P. Dreesen, K. Batselier, and B. De Moor, "Multidimensional realisation theory and polynomial system solving," International Journal of Control, vol. 91, no. 12, pp. 2692-2704, 2018.

[10] M. G. Frost and C. Storey, "Equivalence of a matrix over $R[s, z]$ with its Smith form," International Journal of Control, vol. 28, no. 5, pp. 665-671, 1979.

[11] M. G. Frost and M. S. Boudellioua, "Some further results concerning matrices with elements in a polynomial ring," International Journal of Control, vol. 43, no. 5, pp. 1543-1555, 1986.

[12] Z. Lin, M. S. Boudellioua, and L. Xu, "On the equivalence and factorization of multivariate polynomial matrices," in Proceedings of the 2006 IEEE International Symposium on Circuits 
and Systems, vol. 54, pp. 4911-4914, IEEE, Island of Kos, Greece, May 2006.

[13] M. S. Boudellioua, "Further results on the equivalence to Smith form of multivariate polynomial matrices," Control and Cybernetics, vol. 42, no. 2, pp. 543-551, 2013.

[14] M. S. Boudellioua, "Computation of the Smith form for multivariate polynomial matrices using Maple," American Journal of Computational Mathematics, vol. 2, no. 1, pp. 21-26, 2012.

[15] T. Cluzeau and A. Quadrat, "Factoring and decomposing a class of linear functional systems," Linear Algebra and Its Applications, vol. 428, no. 1, pp. 324-381, 2008.

[16] M. S. Boudellioua and A. Quadrat, "Serre's reduction of linear function systems," Mathematics in Computer Science, vol. 4, no. 2-3, pp. 289-312, 2010.

[17] D. Li, J. Liu, and L. Zheng, "On the equivalence of multivariate polynomial matrices," Multidimensional Systems and Signal Processing, vol. 28, no. 1, pp. 225-235, 2017.

[18] J. Liu, D. Li, and L. Zheng, "Minor prime factorization for $n-\mathrm{D}$ polynomial matrices over arbitrary coefficient field," Complexity, vol. 2018, Article ID 6235649, 9 pages, 2018.

[19] D. Quillen, "Projective modules over polynomial rings," Inventiones Mathematicae, vol. 36, no. 1, pp. 167-171, 1976.

[20] D. Li, J. Liu, and D. Chu, "The Smith form of a multivariate polynomial matrix over an arbitrary coefficient field," Linear and Multilinear Algebra, pp. 1-14, 2020.

[21] M. Wang and D. Feng, "On Lin-Bose problem," Linear Algebra and its Application, vol. 390, pp. 279-285, 2004. 